Supporting Information

\title{
Strain rate dependent shear plasticity in graphite oxide
}

Soumya Vinod ${ }^{1}+$, Chandra Sekhar Tiwary $^{1}{ }^{*}$, Leonardo D. Machado ${ }^{2}+$, Sehmus Ozden $^{1}$, Juny Cho ${ }^{1}$, Preston Shaw ${ }^{1}$, Robert Vajtai ${ }^{1}$, Douglas S. Galvão ${ }^{2 *}$, Pulickel M. Ajayan ${ }^{1 *}$

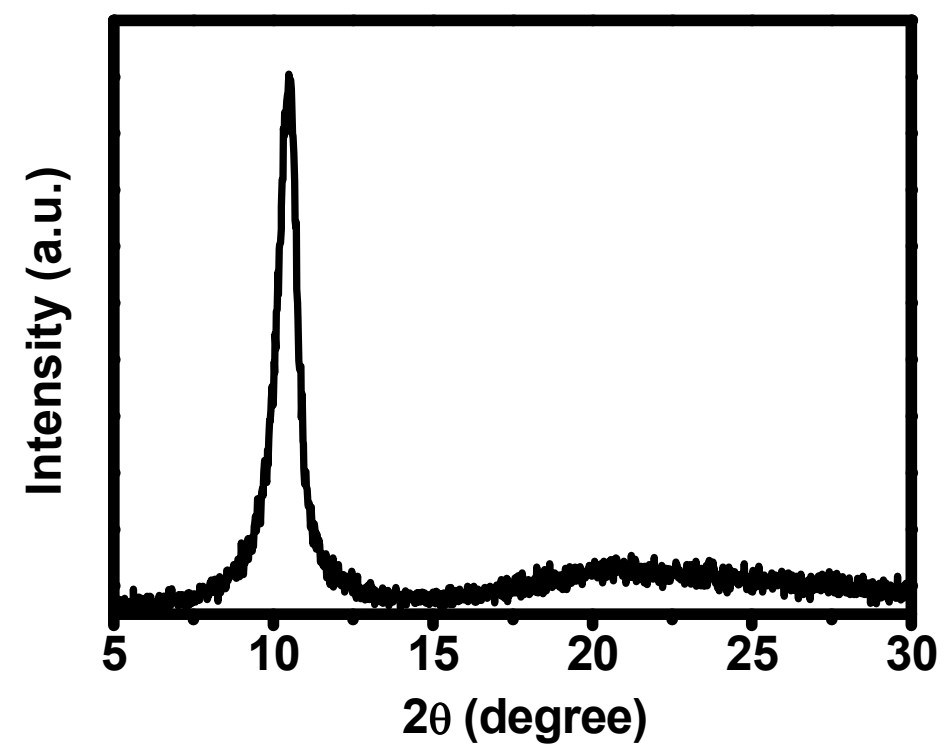

Fig. S1.X-ray diffractionof GO paper. XRD data showing a peak at $\sim 10$ degrees corresponding to interlayer spacing of $0.8 \mathrm{~nm}$ due to the presence of functional groups and confinement of water molecules between the layers 

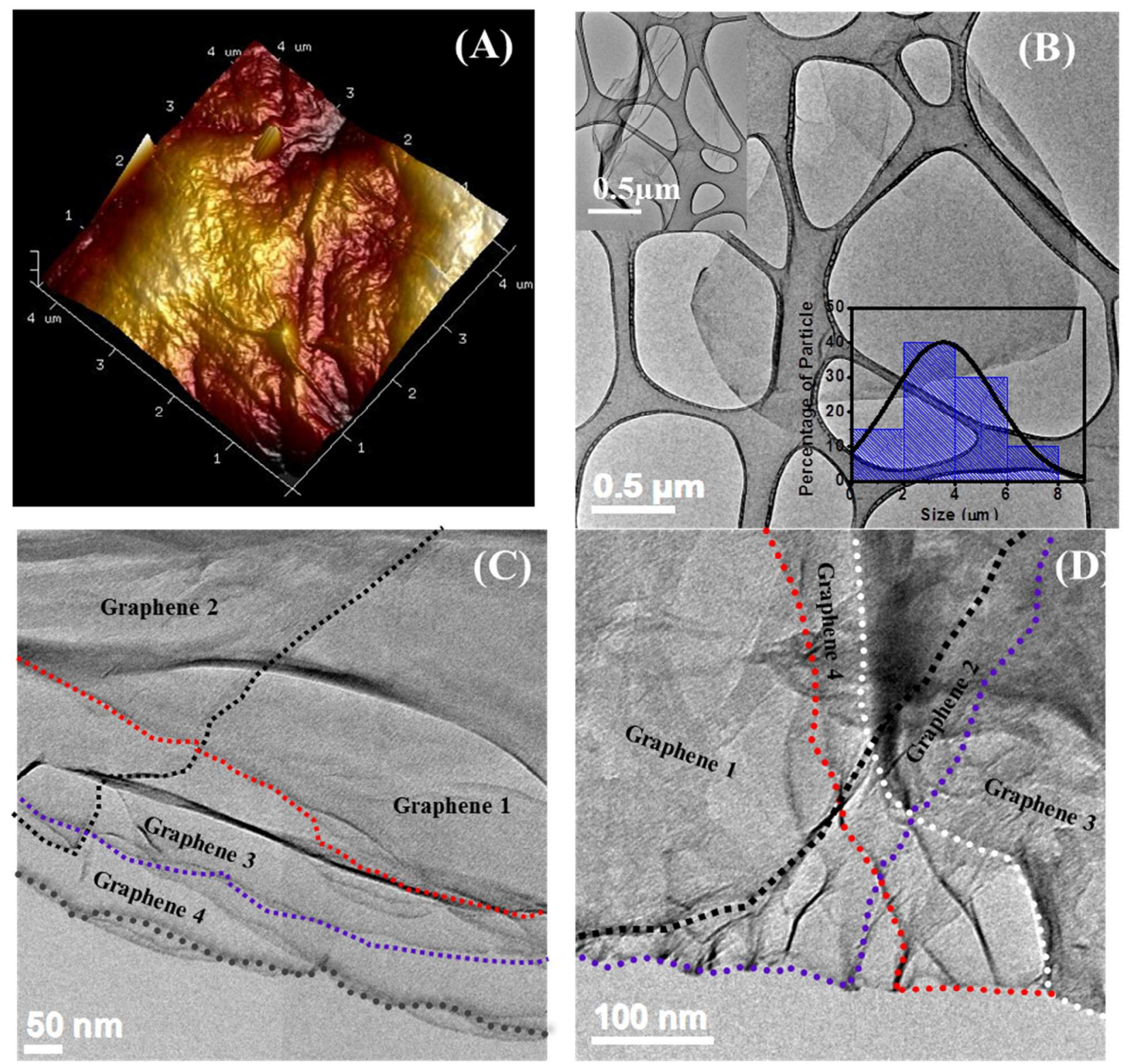

Fig. S2: Structural characterization of GO paper. (A) AFM showing the presence of wrinkles in individual GO sheets due to the defects and functional groups along with the overlap of different sheets causing the rugged surface of the paper. TEM of (B) initial GO sheet with size distribution shown in inlay. (C-D)shows multiple GO sheets randomly stacked on top of each other in different ways. These GO sheets are oriented in different directions and the large area film is formed by the overlapping of these sheets 

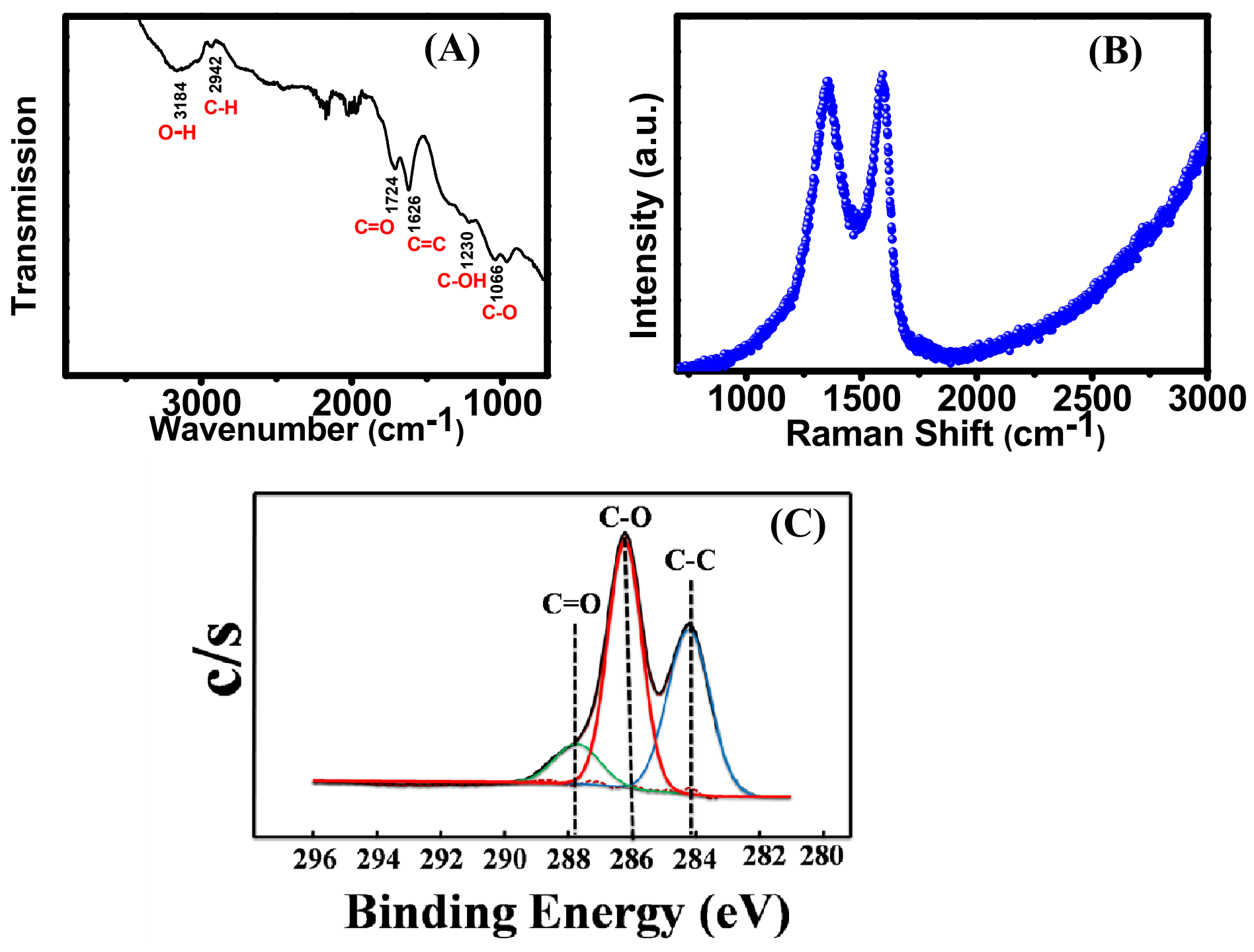

Fig. S3: Spectroscopic characterization of GO paper. (A) Chemical bonds GO paper were identified using FTIR andthe functional groups in GO such as hydroxyl, carboxyl and epoxide were observed. (B) Raman shows the $\mathrm{G}$ band at $1600 \mathrm{~cm}^{-1}$ which arises from the $\mathrm{sp}^{2}$ hybridized carbon atoms and $\mathrm{D}$ band located at $1350 \mathrm{~cm}^{-1}$ is from the disorder created from the different functional groups present in GO. (C) XPS analysis was used to determine detail surface characterization of $\mathrm{GO}$ paper. It consists of $65.9 \% \mathrm{C}$ and $34.1 \% \mathrm{O}$ atoms. The $\mathrm{C} 1 \mathrm{~s}$ core level of peak position of carbon atom is approximately at $285 \mathrm{eV}$. In high-resolution XPS analysis, the C$\mathrm{C}$ and $\mathrm{C}-\mathrm{O}$ bonds are at 284.2 and $286.23 \mathrm{eV}$. The peak at 287.6 corresponds to $\mathrm{C}=\mathrm{O}$ bonding. 


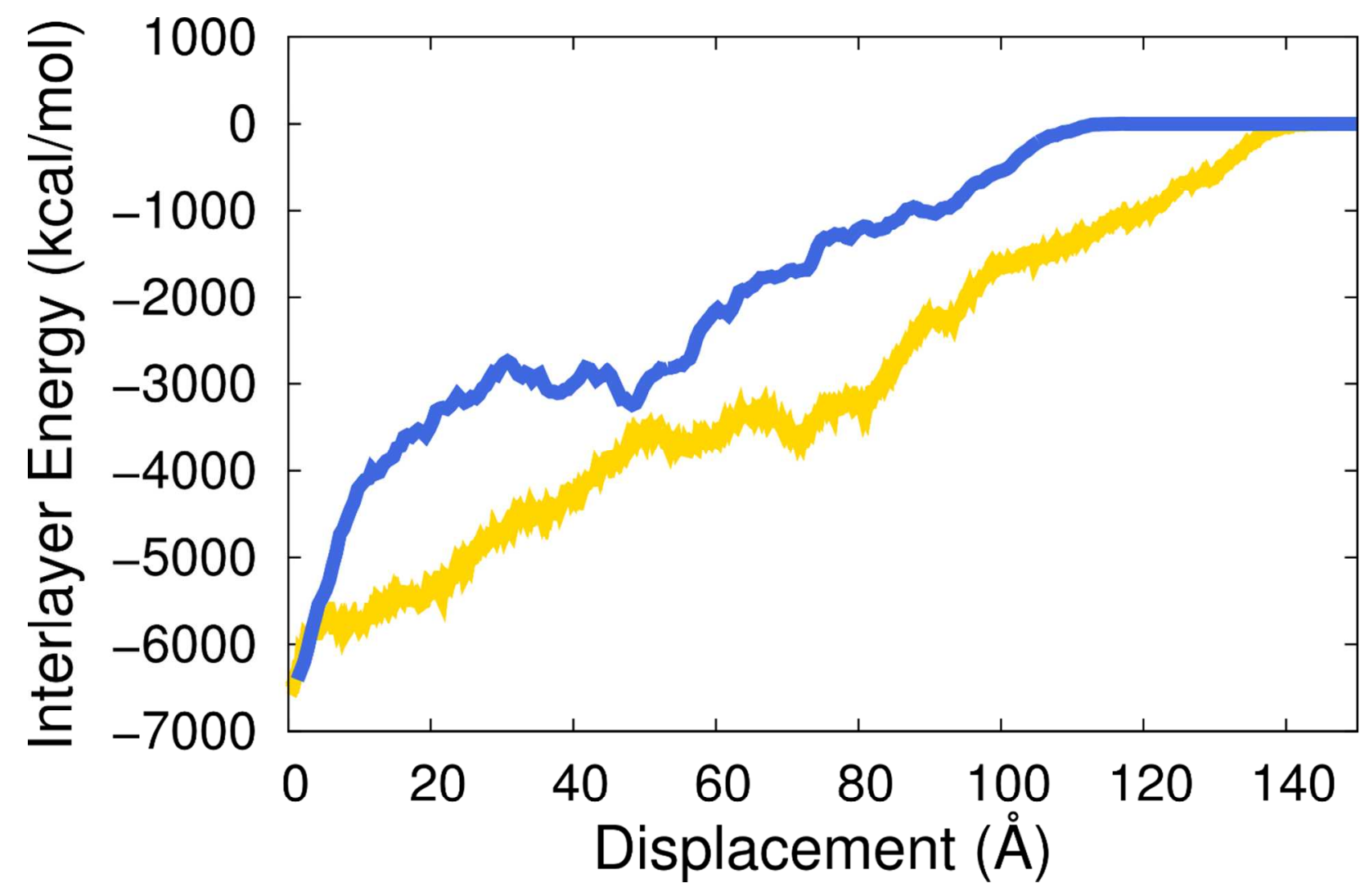

Fig. S4:Interaction energy between the top and adjacent layers, for high (blue) and low (yellow) strain rates. Although the energy landscape roughness corresponds to small changes in energy, these are caused by small displacements, resulting in large forces. Notice that the total change in energy is the same regardless of the pulling rate, since the initial configuration is the same and in the final configuration the top and adjacent layers are completely separated. 

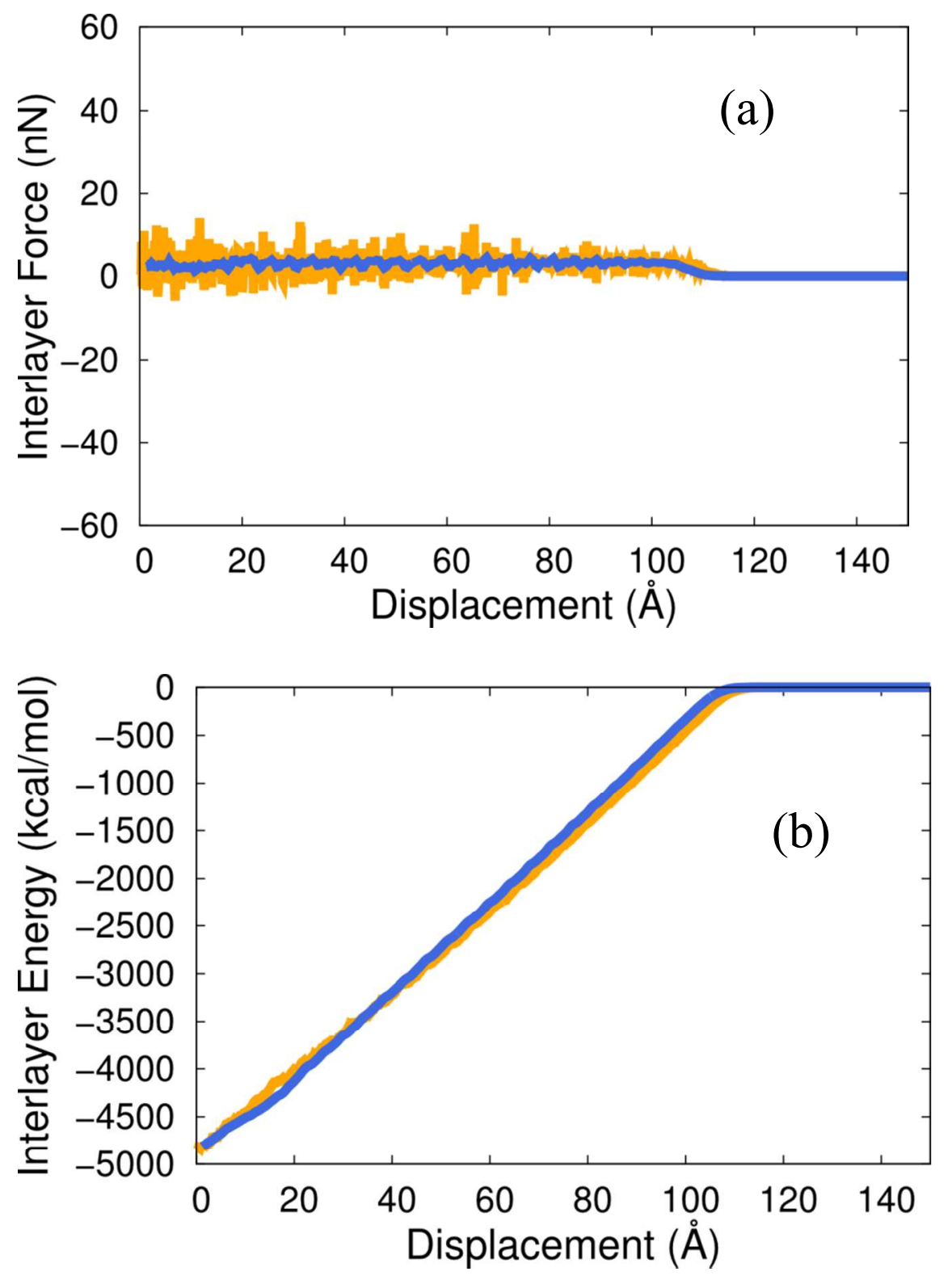

Fig. S5: Interlayer Force and Interaction energy between the top and adjacent layers, for high (blue) and low (yellow) strain rates for graphene. A comparison video (Movie S3) of slow strain rate is shown as compared to GO. No change is observed in the both layers behavior 